\title{
Mechanochemical Energy Transduction during the Main Rotary Step in the Synthesis Cycle of $F_{1}$-ATPase
}

\author{
Jacek Czub, ${ }^{\dagger}$ Miłosz Wieczór, ${ }^{\dagger}$ Bartosz Prokopowicz, ${ }^{\dagger}$ and Helmut Grubmüller*, \\ ${ }^{\dagger}$ Department of Physical Chemistry, Gdansk University of Technology, ul. Narutowicza 11/12, 80-233 Gdańsk, Poland \\ ${ }^{\ddagger}$ Department of Theoretical and Computational Biophysics, Max Planck Institute for Biophysical Chemistry, Am Fassberg 11, 37077 \\ Göttingen, Germany
}

Supporting Information

ABSTRACT: $\mathrm{F}_{1}$-ATPase is a highly efficient molecular motor that can synthesize ATP driven by a mechanical torque. Its ability to function reversibly in either direction requires tight mechanochemical coupling between the catalytic domain and the rotating central shaft, as well as temporal control of substrate binding and product release. Despite great efforts and significant progress, the molecular details of this synchronized and fine-tuned energy conversion mechanism are not fully understood. Here, we use extensive molecular dynamics simulations to reconcile recent single-molecule experiments with structural data and provide a consistent thermodynamic, kinetic and mechanistic description of the main rotary substep in the synthetic cycle of mammalian ATP synthase. The

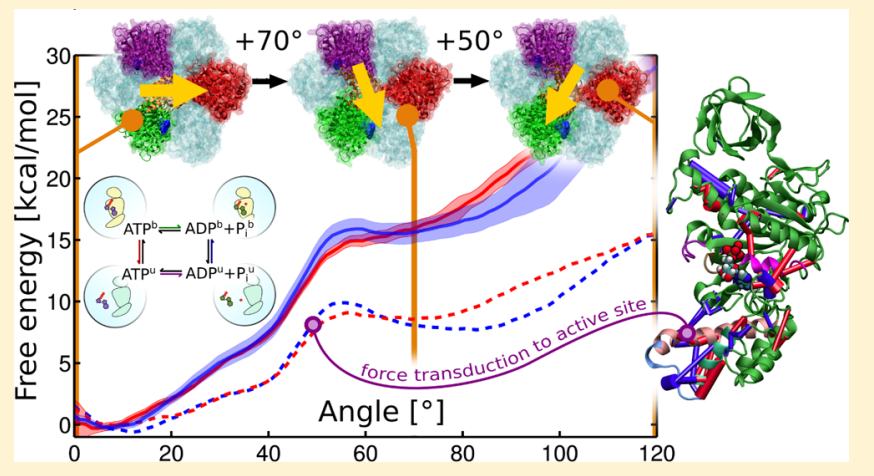
calculated free energy profiles capture a discrete pattern in the rotation of the central $\gamma$-shaft, with a metastable intermediate located-consistently with recent experimental findings-at $70^{\circ}$ relative to the X-ray position. We identify this rotary step as the ATP-dependent substep, and find that the associated free energy input supports the mechanism involving concurrent nucleotide binding and release. During the main substep, our simulations show no significant opening of the ATP-bound $\beta$ subunit; instead, we observe that mechanical energy is transmitted to its nucleotide binding site, thus lowering the affinity for ATP. Simultaneously, the empty subunit assumes a conformation that enables the enzyme to harness the free energy of ADP binding to drive ATP release. Finally, we show that ligand exchange is regulated by a checkpoint mechanism, an apparent prerequisite for high efficiency in protein nanomotors.

\section{INTRODUCTION}

ATP synthase is a ubiquitous, evolutionarily conserved enzyme that reversibly couples the synthesis or hydrolysis of ATP to the electrochemical proton gradients across energy-transducing membranes in mitochondria, chloroplasts and bacteria. ${ }^{1-3}$ It consists of two opposing rotary motors, the membraneembedded, ion-translocating $\mathrm{F}_{\mathrm{o}}$ and hydrophilic, ATP-driven $\mathrm{F}_{1}$ (Figure 1A), mechanically coupled by a common rotor. In the synthesis mode, when the proton motive force exceeds the chemical potential of ATP hydrolysis, the rotation of $F_{o}$, powered by proton translocation, induces the stepwise rotary motion of the asymmetric $\gamma$ subunit $\left(\gamma\right.$-shaft) of $\mathrm{F}_{1}$-ATPase inside the hexameric cylinder comprised of three alternating $\alpha$ and $\beta$ subunits $\left(\alpha_{3} \beta_{3}\right.$ hexamer). In response to the $\gamma$-shaft rotation, the three catalytic sites located mostly on the $\beta$ subunits undergo coordinated transitions between conformational states differing in their affinity for nucleotide ligands. According to this binding change mechanism, the cyclic conformational changes of $\beta$ subunits drive net synthesis of ATP from ADP and inorganic phosphate $\left(\mathrm{P}_{i}\right)$ even under physiological conditions that strongly favor the hydrolysis reaction. $1,2,4,5$
Single-molecule rotation imaging has shown that each hydrolysis and synthesis cycle of $\mathrm{F}_{1}$-ATPase is accompanied by a $120^{\circ}$ step of the $\gamma$-shaft. ${ }^{6-9}$ In the bacterial $\mathrm{F}_{1}$-ATPase, these $120^{\circ}$ steps were further resolved into $80^{\circ}$ and $40^{\circ}$ rotary substeps, ${ }^{10,11}$ which, in the hydrolysis direction, follow the ATP-waiting pause and the catalytic pause, respectively. More recently, it has been found that the mitochondrial form of the enzyme displays a more complex stepping pattern with three successive substeps: the main $65^{\circ}$ step triggered by ATP binding and two shorter $25^{\circ}$ and $30^{\circ}$ steps induced by $\mathrm{P}_{i}$ release and hydrolysis reaction, respectively. ${ }^{12,13}$ Remarkably, unlike in most linear motors ${ }^{14}$ or helicases, ${ }^{15}$ the mechanochemical energy conversion during these steps is almost $100 \%$ efficient, as found by measuring the mechanical work done by the rotating $\gamma$-shaft against a viscous $\operatorname{drag}^{7}$ and a conservative external force. $^{16}$

Despite numerous efforts, ${ }^{2,3,17-24}$ the structural determinants of the free-energy transduction by ATP synthase are still only partly understood. It has been suggested that elastic power

Received: November 16, 2016

Published: March 2, 2017 

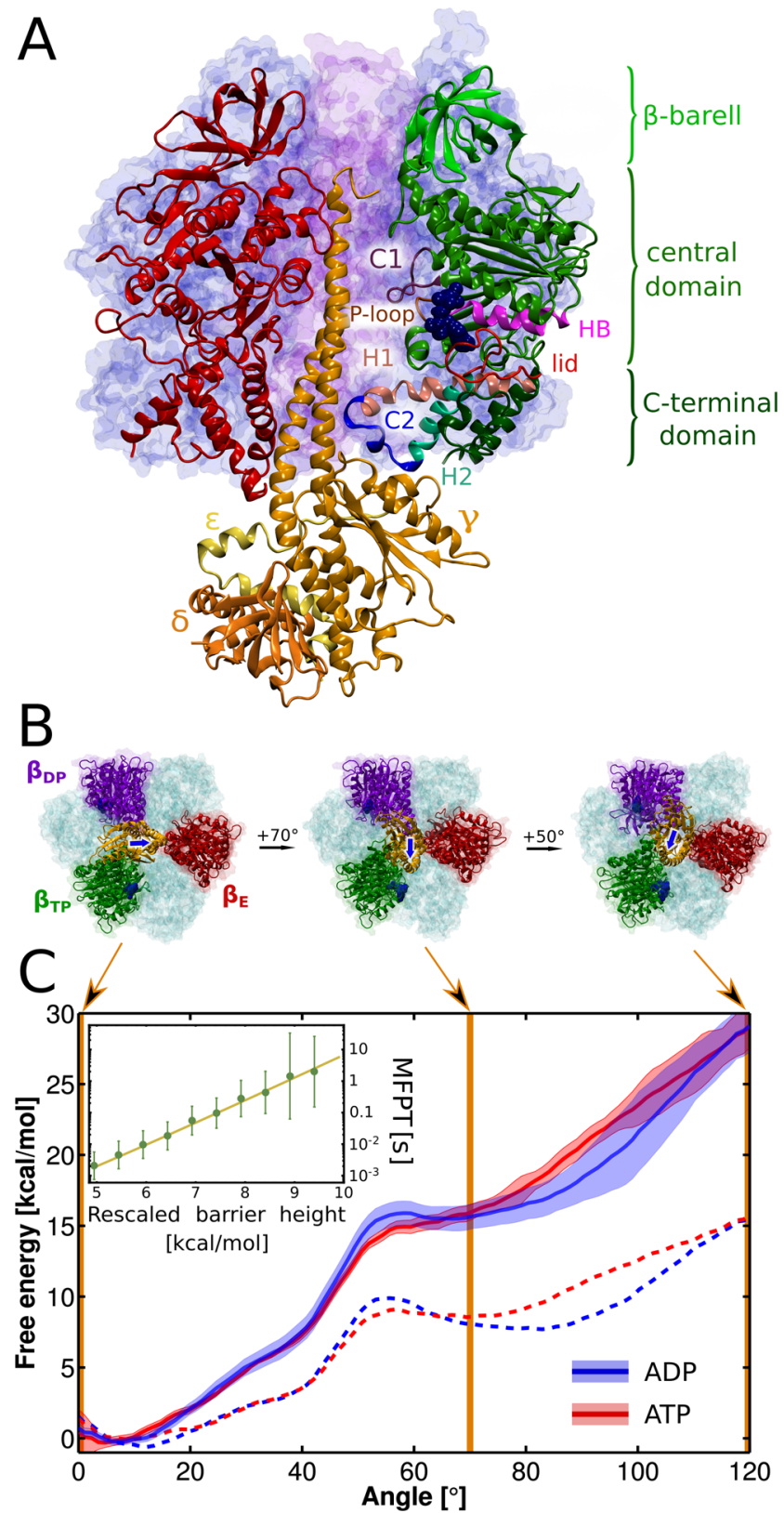

energy of rotating $\gamma$ subunit is converted into conformational energy of the $\alpha_{3} \beta_{3}$ subcomplex and harnessed to drive the changes in occupancy of the nucleotide-binding sites is much less clear. In particular, little is known about the $\gamma$-mediated allosteric communication between the catalytic subunits that leads to a well-recognized anticooperativity of the binding sites which is important for rapid catalysis. ${ }^{30,31}$

On the basis of structural and nucleotide-binding data, it has long been assumed that the rotating $\gamma$-shaft causes the catalytic $\beta$ subunits to cycle between the high-affinity closed state and the low-affinity open state and that the main cost of the synthetic cycle is associated with ATP release. ${ }^{2,4,32}$ Indeed, by combining single-molecule FRET with rotation imaging it has been shown that the open-to-closed transitions are synchronized with the motion of $\gamma$ and occur in three stages with the intermediate partially closed conformation of $\beta$ observed in the ATP-waiting pause. ${ }^{33}$ Further, a typical crystal structure containing two closed $\left(\beta_{\mathrm{DP}}\right.$ and $\beta_{\mathrm{TP}}$ in Figure 1A) and open $\left(\beta_{\mathrm{E}}\right)$ catalytic subunit represents either catalytic or the pre- $\mathrm{P}_{i^{-}}$ release state. ${ }^{12,23}$ Additional residue-specific insight was provided by several mutagenesis studies which identified regions critical for the enzyme's mechanochemistry. In particular, the catch 2 loop and Arg408 in helix 2 of the Cterminal domain were found to be essential for synchronizing the $\gamma$-shaft rotation with the open-to-closed transition of $\beta,{ }^{34,35}$ while the hinge region at the $\mathrm{N}$-terminal end of helix $\mathrm{B}$ was shown to be involved in coupling the ligand binding to the conformational change (Figure 1A). ${ }^{36,37}$ Furthermore, a number of residues in the phosphate-binding P-loop motif and in the catch 1 loop (Asp316 and Asp319) are important for the proper recognition and binding of nucleotides in the catalytic site. $^{34,38}$

In recent years, early semiquantitative static models of $\mathrm{F}_{1^{-}}$ ATPase cycle ${ }^{17}$ were tested and further developed by means of molecular simulations. ${ }^{19,22,23,39-41}$ By including a detailed description of conformational energetics and thermally activated dynamics, this approach allowed for explaining some of the aspects of the free energy transduction mechanism at the atomic level. Specifically, a nonequilibrium response of the $\alpha_{3} \beta_{3}$ conformation to the rotary motion of the $\gamma$-shaft was examined through enforced-rotation molecular dynamics (MD) simulations, providing an initial insight into the energy transmission in the synthesis direction. ${ }^{19,42}$ The nucleotide-dependent conformational transitions of the $\alpha_{3} \beta_{3}$ ring in the hydrolysis mode were studied through targeted MD and nonequilibrium force-probe simulations, ${ }^{40,41}$ while intrasubunit determinants of these transitions were analyzed in detail by free energy simulations of the isolated $\beta .^{42,43}$ Simulations also helped to elucidate the timing of $\mathrm{P}_{i}$ release in the hydrolytic cycle by providing appropriate angle-dependent dissociation free energies. ${ }^{23}$ Importantly, the first conformational/chemical free energy landscape for the entire rotary cycle has also been determined using the coarse-grained model of $F_{1}$ and an interpolation scheme for the conformational transitions of the binding sites. ${ }^{22,24,44}$ This landscape offered a complete structure-based thermodynamic description of the mechanochemical coupling in $\mathrm{F}_{1}$ and reproduced the stepwise rotation of the $\gamma$-shaft with two substeps between the stable positions. ${ }^{22}$ Additionally, this approach yielded the $F_{1}$-generated torque value consistent with experimental findings ${ }^{24,45}$ and predicted an electrostatic rather than steric origin of the energy conversion mechanism. $^{22,24}$ It should be also noted that recently the sequence of conformational transitions underlying 
the mechanism of a related hexameric $V_{1}$-ATPase has been investigated in detail using free energy simulations. ${ }^{46}$

Here, to understand the mechanochemistry of $F_{1}$-ATPase in structural and energetic terms at atomic resolution, we have carried out extensive umbrella sampling MD simulations, with accumulated simulation time of $10 \mu \mathrm{s}$, to determine the free energy profile for the rotation of the $\gamma$-shaft inside the $\alpha_{3} \beta_{3}$ hexamer. The resulting profile is consistent with the stepwise character of rotation in synthesis direction, with the main $70^{\circ}$ substep occurring before the ATP-dependent metastable state, in agreement with the single-molecule data. ${ }^{12}$ Further, we find that the major mechanical energy input for ATP synthesis is provided during this $70^{\circ}$ substep and that it is fine-tuned to the thermodynamic cost of the following nucleotide exchange under physiological conditions. Our simulations also provide structural insight into the mechanism through which the energy of rotating $\gamma$ is used to drive the occupancy change of the nucleotide binding sites. In particular, we explain the determinants of stalling the rotation at $70^{\circ}$ and propose its role in harnessing the energy gain due to $\mathrm{ADP}$ binding to induce ATP release.

\section{RESULTS AND DISCUSSION}

Free Energy Simulations Accurately Capture the Metastable Intermediate in the Synthesis Cycle of $F_{1}$ ATPase. To understand structural determinants of the mechanochemical energy transfer during the $F_{1}$ rotary synthesis, we first computed the free energy profile for the rotation of the $\gamma$-shaft within the $\alpha_{3} \beta_{3}$ ring by $120^{\circ}$, starting from its angular position captured in the X-ray structure (Figure 1A). To this end, we used umbrella sampling with initial configurations obtained from a $1 \mu \mathrm{s}$ flexible-axis rotation simulation (see Methods). In this simulation an external torque, mimicking the effect of $\mathrm{F}_{\mathrm{o}}$, was used to drive the rotation of the $\gamma$-shaft from the initial position $\left(0^{\circ}\right)$ with its convex side facing the $\beta_{\mathrm{E}}$ subunit (empty) to the final position $\left(+120^{\circ}\right)$ facing the $\beta_{\mathrm{TP}}$ subunit (nucleotide-bound) (Figure $1 \mathrm{~B}$ and Movie S1 and Movie S2 in SI). The applied angular velocity of $0.12^{\circ} / \mathrm{ns}$ is by a factor of 100 faster than the instantaneous speed of the $\gamma$ subunit measured in single-molecule experiments under external load, ${ }^{2,47}$ but is in fact of the same order of magnitude as the maximum rotational speed without external load estimated by Okazaki and Hummer. ${ }^{29}$

The resulting profile shown in Figure 1C (blue solid line) reveals that initially the free energy rises steeply up to $16 \pm 0.8$ $\mathrm{kcal} / \mathrm{mol}$ while the gamma shaft rotates from 0 to ca. $60^{\circ}$. Clearly, this free energy barrier stabilizes the $\gamma$-shaft around its $\mathrm{X}$-ray structure position (close to $0^{\circ}$ ) and explains why external torque applied by the $c$-ring is necessary for the $F_{1}$ motor to proceed in synthesis direction at the speed observed under physiological conditions (ATP synthesis rate of $60-80 \mathrm{~s}^{-1}$ ). ${ }^{32}$ After $\sim 60^{\circ}$ the free energy levels off and exhibits a shallow minimum at $\sim 70^{\circ}$, which might be indicative of a metastable state of the motor. Notably, in this region, our free energy profile is probably not fully converged, with the minimum showing a tendency to get deeper with increasing sampling (see Figure S1). Consequently, the free energy around $\sim 70^{\circ}$ might in fact be lower by ca. $3.5 \mathrm{kcal} / \mathrm{mol}$, as shown below by comparison with thermodynamic data.

From single-molecule experiments, it has been recently concluded that the $120^{\circ}$ hydrolysis cycle of mitochondrial $F_{1}$ ATPase proceeds in three successive rotary substeps: the $65^{\circ}$ step following ATP binding to one $\beta$ subunit, the $25^{\circ}$ step triggered by $\mathrm{P}_{i}$ release from another $\beta$ subunit, and the $30^{\circ}$ step induced by the hydrolysis event at the third $\beta$ subunit. ${ }^{12}$ The presence of a second minimum in our free energy profile is consistent with this finding and strongly suggests that the rotation in the synthesis direction also displays a discrete pattern. This is even more evident when the $\mathrm{F}_{\mathrm{o}}$-generated torque potential with a constant slope of $-0.1 \mathrm{kcal} /{ }^{\circ}$ (corresponding to the energy input of $12.5 \mathrm{kcal} / \mathrm{mol}$ per synthetic cycle ${ }^{48}$ ) is added to the free energy profile, yielding a well-pronounced minimum implying the resting position of the $\gamma$-shaft (dashed blue line). Importantly, the location of the second stable position at $+70^{\circ}$ with respect to the $\mathrm{X}$-ray structure confirms the previous hypothesis ${ }^{12}$ that the canonical $\mathrm{F}_{1} \mathrm{X}$-ray structures (e.g., 1BMF, 1E79, 2JIZ) $)^{4,49}$ represent the pre- $\mathrm{P}_{i}$ release state. Accordingly, the second minimum revealed by our profile corresponds to the ATP-dependent state, which in synthesis direction should precede ATP release from $\beta_{\mathrm{TP}}$. Because the release of ATP from the low-affinity $\beta$ occurs on a millisecond time scale ${ }^{32}$ it is, unsurprisingly, not observed in our microsecond simulations. Consequently, the free energy increase in the $80-120^{\circ}$ range corresponds to the rotation against the nucleotide-containing $\beta_{\mathrm{TP}}$ and therefore it is presumably responsible for stalling the rotary motion of the $\gamma$-shaft until ATP is released. Note also that because of the fixed occupancy of the binding sites, the free energy curve corrected by the torque potential cannot approach 0 at $120^{\circ}$, which could be expected at longer time scales allowing for binding/ unbinding events to occur.

To check if the presence of the second minimum in the free energy profile depends on the type of nucleotide molecule bound to the $\beta_{\mathrm{TP}}$ subunit, we repeated the above calculations after replacing the $\beta_{\mathrm{TP}}$-bound $\mathrm{ADP}$, present in the $\mathrm{X}$-ray structure, with ATP, by gradually transforming one nucleotide into the other over $300 \mathrm{~ns}$ (see Methods). Figure 1C shows that the profiles obtained in the presence of ATP (red lines) do not markedly differ from the original ones, indicating that the type of nucleotide occupying the $\beta_{\mathrm{TP}}$ subunit does not change the rotation pattern. In fact, the free energy required to rotate the $\gamma$-shaft in the presence of ATP is only slightly higher (up to $2.5 \mathrm{kcal} / \mathrm{mol}$ within the second minimum), which is in qualitative agreement with the nucleotide-binding data ${ }^{32}$ showing that at this stage of the cycle ATP binds to $\beta$ with $3.9 \mathrm{kcal} / \mathrm{mol}$ higher affinity than ADP. Consequently, the structure and dynamics of ATP- and ADP-bound $\beta$ subunits are quite similar, as can be seen from the distribution of $80 \mathrm{X}$-ray $\beta$ conformations and the MD-generated conformational ensembles (Figure S2). Thus, our free energy profiles in Figure 1C suggest that the energetics of the $\gamma$-shaft rotation is in fact dominated by the coupling to the conformational state of the $\alpha_{3} \beta_{3}$ subcomplex and hence depends to a much larger extent on the conformation than the occupancy of the $\beta_{\mathrm{TP}}$ subunit.

Thermodynamics of the Main Rotary Substep Suggest That ATP Release Requires Concurrent ADP Binding. It is well-established that ATP binding provides the main energy input for the enzyme operating in hydrolysis direction, driving the longest substep in the catalytic cycle (by $\sim 65^{\circ}$ and $\sim 80^{\circ}$ in the mammalian and bacterial variant of $F_{1}$, respectively). ${ }^{2,12}$ Accordingly, the free energy increase in the range $0 \rightarrow 70^{\circ}$ (Figure $1 \mathrm{C}$ ) is compatible with the idea that it is also this substep of the synthetic cycle where the main (mechanical) energy input for ATP synthesis is provided. The exceptionally high efficiency of ATP synthase ${ }^{16}$ implies that this energy input should be roughly equal to the total free energetic 
cost of the binding site occupancy change following the $0 \rightarrow$ $70^{\circ}$ substep. In other words, a progression along the motor's "chemical" coordinate occurs once the free energy accumulated in the structure by the rotating $\gamma$ suffices to drive the occupancy change. According to single-molecule experiments, ${ }^{12}$ this occupancy change involves ATP release from $\beta_{\mathrm{TP}}$ and, presumably, also ADP binding to $\beta_{\mathrm{E}}$. The thermodynamic cost of ATP to ADP exchange, $\Delta G_{\text {occ }}$, can be estimated to be $12.0 \mathrm{kcal} / \mathrm{mol}$ (see SI Methods and Figure S3 for a corresponding thermodynamic cycle, an approach that has been previously shown to greatly simplify the analysis of energy transduction in protein motors). ${ }^{39,44}$ This estimate is also consistent with the exchange cost of ca. $13 \mathrm{kcal} / \mathrm{mol}$, obtained from the dependence of the affinities for ATP and ADP on the $\gamma$ angular position. ${ }^{50}$ The free energy profile in Figure $1 \mathrm{C}-$ obtained without any fitting parameters-shows that the above values agree quite well with the reversible work required to rotate $\gamma$ by $70^{\circ}$ to the putative pre-ATP-release minimum, $\Delta G_{\text {rot }}=15.5 \pm 0.9 \mathrm{kcal} / \mathrm{mol}$. This also indicates that in the least converged region of our free energy profile (Figure S1) the error does not exceed $3.5 \mathrm{kcal} / \mathrm{mol}$.

The good agreement between these values suggests that the rotation work is in fact fine-tuned to the cost of ligand exchange under physiological conditions and supports the picture according to which ATP release from $\beta_{\mathrm{TP}}$ and ADP binding to $\beta_{\mathrm{E}}$ occur at the angle of $\mathrm{ca} .+70^{\circ}$ with respect to the $\mathrm{X}$-ray structure position. ${ }^{50}$ An important consequence is also that this product release is only partially driven by the torque transmitted by the $\gamma$ subunit; the remaining free energy is provided through $\mathrm{ADP}$ binding at a remote site $\left(\beta_{\mathrm{E}}\right)$. This conclusion also underscores that the allosteric coupling between the three beta subunits via the gamma subunit is essential for the function of the enzyme.

To additionally test the above conclusions, we used our simulation data to examine the kinetics of the main rotary substep in the ATP synthesis cycle $\left(0^{\circ} \rightarrow 70^{\circ}\right)$. To this end, the free energy profiles, tilted by the $\mathrm{F}_{\mathrm{o}}$-generated torque potential (dashed lines in Figure 1C) were used to simulate the $\gamma$-shaft rotary substep using Brownian dynamics (BD) (see SI Methods). With this approach, we computed the dependence of the mean first passage time (MFPT) of the $\gamma$-shaft from $0^{\circ}$ to $70^{\circ}$ as a function of the activation barrier by running the BD simulations independently for a set of free energy profiles scaled by a constant factor (see SI Methods), using an angledependent diffusion coefficient obtained from our atomistic simulations (see Figure S4). Similar dependence was also calculated from the transition state theory (Figure 1C, inset). We found that for our MFPT estimate to be consistent with the experimental rotation time scale $(\sim 0.010-0.015 \mathrm{~s}))^{32}$ the barrier needs to be lowered by $3.5-4.0 \mathrm{kcal} / \mathrm{mol}$ (Figure 1C, inset). This might result in part from the above-mentioned incomplete relaxation of the system but is also indicative of the fact that that precise prediction of the rotation times would require explicit consideration of the conformational coordinate of the motor. 22

$\gamma$-Shaft Rotation Alone Does Not Trigger the Opening of ATP-Bound $\beta$ Subunit. Next, to understand the structural basis for the efficient energy conversion during the main rotary substep, we examined conformational response of the catalytic portion of $\mathrm{F}_{1}$ to $\gamma$-shaft rotation. For that purpose, we computed the extent of the opening/closing of the three $\beta$ subunits in response to the $\gamma$-shaft progression, using the position along the vector connecting the open and closed states in the conformational space as the collective coordinate (see Conformational coordinate 1 in Figure S2). Figure 2A shows

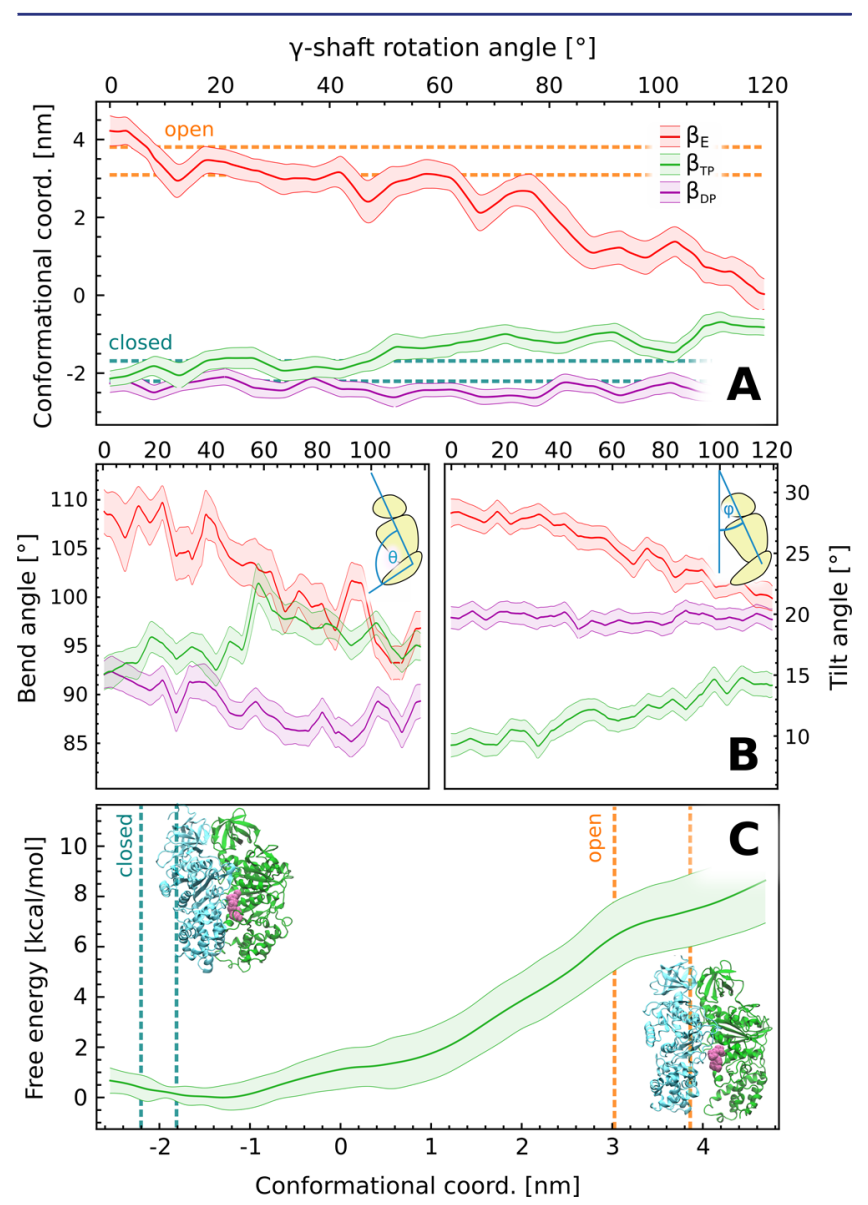

Figure 2. (A) Conformational response of the three $\beta$ subunits to the $\gamma$-shaft rotation. The main conformational coordinate describing the opening/closing conformational change is defined as the vector connecting the open and closed conformations of $\beta$ present in the $\mathrm{X}$ ray structure. Dashed lines show the extent of fluctuations $( \pm \sigma)$ of the conformational coordinate in equilibrium simulations. (B) Rotationinduced changes in bend (left) and tilt (right) angles that characterize the opening/closing transition. (C) Free energy profile along the main conformational coordinate for an isolated $\alpha \beta$ dimer with ATP bound in the active site; the profile shows a strong preference (ca. $6.7 \mathrm{kcal} /$ $\mathrm{mol}$ ) for the closed state if the nucleotide is present. Note that the conformational coordinate reflects a distance in the conformational space of $\beta$, hence the large change by ca. $5-6 \mathrm{~nm}$ (see Figure S2).

that upon rotation the initially open $\mathrm{ADP}$-waiting $\beta$ subunit $\left(\beta_{\mathrm{E}}\right)$ closes spontaneously and, at the second stable position of the $\gamma$-shaft, adopts the intermediate half-open conformation $(4.0 \rightarrow 2.2 \mathrm{~nm})$ previously proposed as the most stable conformation of the nucleotide free $\beta$ subunit. ${ }^{42}$ In our previous simulations, ${ }^{42}$ this spontaneous transition to the halfopen state was found to closely follow the rotating $\gamma$-shaft, and therefore it precedes $\mathrm{ADP}$ binding which is 2 orders of magnitude slower $(\sim 10 \mathrm{~ms}$ at typical ADP concentration, compared to $100 \mu \mathrm{s}$ required for the rotation of $\gamma$ ). This progressive closing transition of $\beta_{\mathrm{E}}$ might explain a gradual increase in the affinity for ADP in response to $\gamma$ rotation found in single-molecule experiments. ${ }^{50}$ Figure $2 \mathrm{~A}$ also shows that the rotation-induced conformational changes of the two initially closed $\beta$ subunits are less pronounced, with $\beta_{\mathrm{TP}}$ opening by up to $20 \%(-2.0 \rightarrow-0.8 \mathrm{~nm})$ and $\beta_{\mathrm{DP}}$ remaining almost intact. 

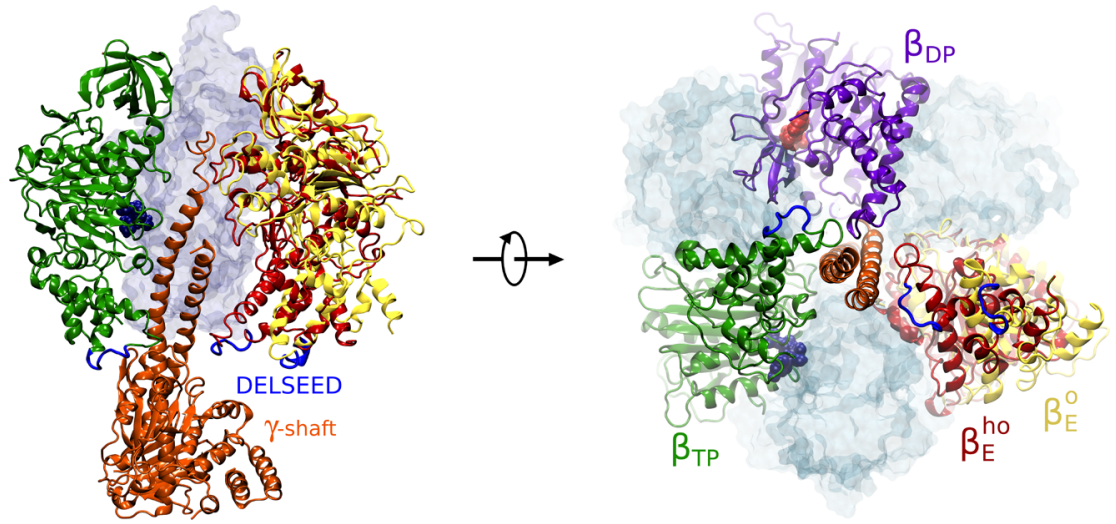

Figure 3. Structure of the $\mathrm{F}_{1}$ motor captured in the intermediate pre-ATP-release state $\left(\right.$ at $\left.70^{\circ}\right)$. An overlay of two conformations of $\beta_{\mathrm{E}}$ is shown: open (in yellow) and half-open (in red). In the half-open state, $\beta_{\mathrm{E}}$ remains in direct contact with $\gamma$, allowing for $\gamma$-mediated coupling of ADP binding by $\beta_{\mathrm{E}}$ and ATP release from $\beta_{\mathrm{TP}}$.

To gain further structural insight into the rotation-induced conformational dynamics of the $\beta$ subunits, we computed the corresponding evolution of the angle between the C-terminal and $\beta$-barrel/central domains (bend angle) and the angle between the entire subunit and the symmetry axis of the $\alpha_{3} \beta_{3}$ hexamer (tilt angle) (Figure 2B). It can be seen that the transition of $\beta_{\mathrm{E}}$ to the half-open state upon the $\gamma$-shaft progression from the first to the second stable position is accompanied by a bending motion of the subunit, as indicated by a $10^{\circ}$ decrease in the bend angle. At the same time, the tilt angle of $\beta_{\mathrm{E}}$ decreases by $\sim 7^{\circ}$ revealing that the lower portion of the subunit slides into the hexamer, as it is no longer forced outward by the convex side of $\gamma$. In contrast, $\beta_{\mathrm{TP}}$ undergoes only partial unbending, with the C-terminal domain rotating by $\sim 7^{\circ}$ with respect to the central domain, while the lower part of the subunit is pushed outward, as reflected by the $\sim 5^{\circ}$ increase in the tilt angle.

Figure 3 shows that, as a result of these conformational motions, in the structure of $\mathrm{F}_{1}$ corresponding to the second minimum at $70^{\circ}$, the $\gamma$ subunit mediates the interaction between the C-terminal domains of $\beta_{\mathrm{E}}$ and $\beta_{\mathrm{TP}}$ by making a contact with the catch 2 loops involving the conserved DELSEED sequence. This specific arrangement suggests a possible mechanism of negative cooperativity between the binding sites that allows to utilize the binding free energy of $\mathrm{ADP}$ to generate a force facilitating ATP release from $\beta_{\mathrm{TP}}$. Particularly, elastic strain energy stored in the half-open $\beta_{\mathrm{E}}$ by ADP binding may be transmitted via the $\gamma$ subunit rotation toward the lower part of $\beta_{\mathrm{TP}}$, thereby contributing to driving ATP unbinding and subsequent opening of the empty $\beta_{\mathrm{TP}}$. Importantly, extracting maximum work from the binding event in this way requires the C-terminal domain of $\beta_{\mathrm{E}}$ to swing toward $\gamma$ prior to $\mathrm{ADP}$ binding, as is indeed observed in our simulations. Otherwise, a substantial fraction of the free energy gain would be dissipated due to a large separation distance between the C-terminal domain $\beta_{\mathrm{E}}$ and $\gamma$ (see the overlaid structures of the open and half-open $\beta_{\mathrm{E}}$ in Figure 3). The above mechanism also explains the well-established importance of the $\gamma$-subunit for allosteric communication between the nucleotidebinding sites, ${ }^{31}$ specifically the fact that $\gamma$-depleted ATPases shows markedly reduced hydrolysis rates, ${ }^{30}$ and accounts for the role of the DELSEED regions in the torque generation upon ATP binding in the hydrolysis cycle. ${ }^{35}$

Free Energy Stored in Allosteric Network Facilitates ATP Release. Elucidating how the mechanical energy of the $\gamma$ - shaft can be used to drive the occupancy change of the binding sites requires to analyze the changes in the conformational energy of the catalytic subunits in response to the $\gamma$-shaft rotation. Since it has been found that a bound nucleotide molecule favors the closed conformation of the $\beta$ subunit, ${ }^{43,51}$ the $20 \%$ opening of $\beta_{\text {TP }}$ seen in Figure 2A suggests that at least part of the energy transmitted by the $\gamma$ rotation might be stored in the opening/closing coordinate of $\beta_{\mathrm{TP}}$. To investigate this possibility, we calculated the changes in the free energy along this coordinate for the isolated $\alpha \beta$ heterodimer with ATP in the active site (see SI Methods for details). The resulting profile in Figure 2C confirms that the ATP-bound state shows a strong preference for the closed conformation, showing a $6.7 \pm 1.8$ $\mathrm{kcal} / \mathrm{mol}$ increase in the free energy upon $\beta$ opening. By subtracting from this increase the previously estimated free energy difference between the open and closed states of the empty $\beta$ subunit $(\sim 0.5 \mathrm{kcal} / \mathrm{mol}),{ }^{42}$ we find that the release of ATP from a closed beta subunit requires $6.2 \pm 2.3 \mathrm{kcal} / \mathrm{mol}$ more free energy than from an open one, which agrees very well with the experimental value $(6.9 \mathrm{kcal} / \mathrm{mol}){ }^{32}$ This agreement strengthens our conclusion that the progression of the $\gamma$-shaft to the second stable position itself does not induce full opening of the ATP-bound $\beta_{\mathrm{TP}}$. Consistently, the free energy profile in Figure $2 \mathrm{C}$ indicates that the partial opening of $\beta_{\mathrm{TP}}$ (up to $-1 \mathrm{~nm}$ ) consumes virtually none of the free energy stored in the structure due to the $\gamma$-shaft rotation $(\sim 15.5 \mathrm{kcal} /$ $\mathrm{mol})$. Therefore, we propose that the actual full opening of $\beta_{\mathrm{TP}}$ in the synthesis cycle occurs only after ATP release which, as mentioned above, possibly requires additional energy input provided by $\mathrm{ADP}$ binding.

Assuming that no significant opening of the $\beta_{\mathrm{TP}}$ subunit takes place during the $70^{\circ}$ rotation, it becomes crucial to identify regions of the catalytic subunit that efficiently transmit and store the conformational energy for later use in the ATP release. To obtain a spatially resolved description of energy transmission and storage within the $\alpha_{3} \beta_{3}$ hexamer during the $\gamma$ shaft rotation, we performed a force distribution analysis (FDA), a procedure designed to identify allosteric networks involved in signal transduction. ${ }^{52}$ Using this approach, we evaluated the changes in inter-residue forces during the two rotary substeps-first from 0 to $70^{\circ}$, and second from 70 to $120^{\circ}$ - against the steep increase in the free energy profile (see Figure 1). To be able to distinguish between different strain propagation pathways, the changes in force distributions were computed using averages in the $0-10^{\circ}$ (X-ray minimum) or 


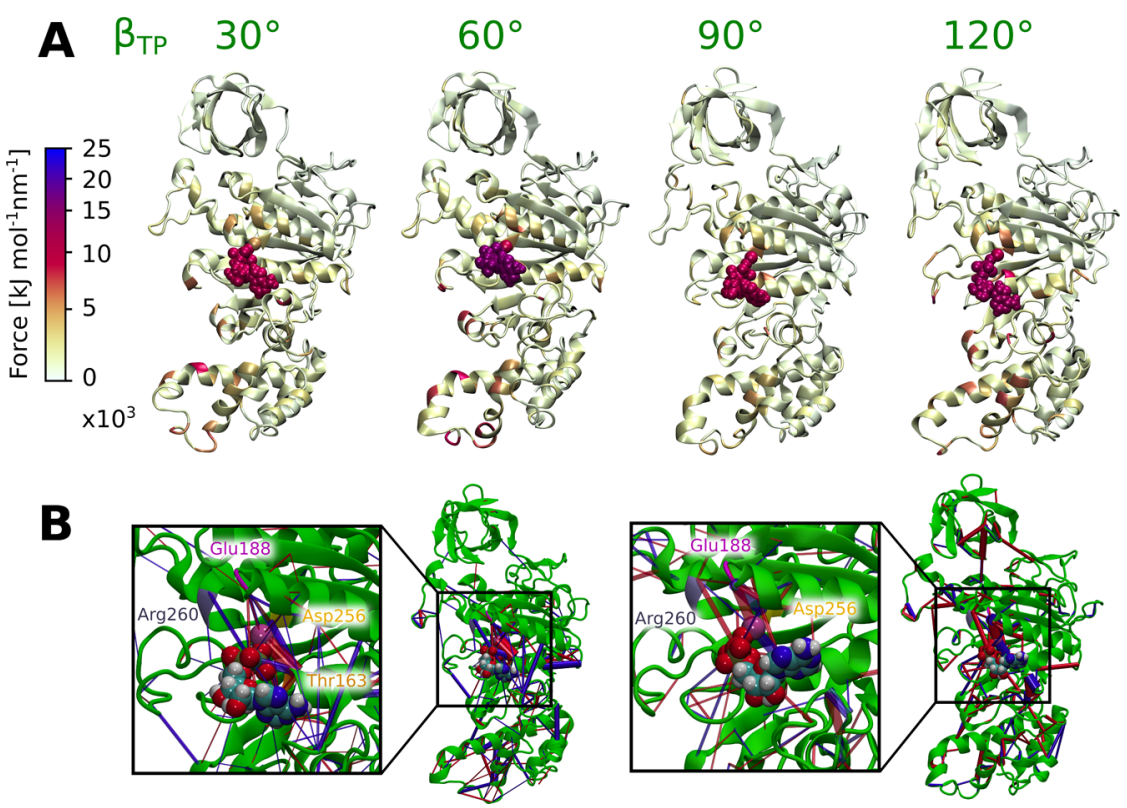

Figure 4. (A) Rotation-induced changes in inter-residue forces, color-mapped onto the structure of $\beta_{\mathrm{TP}}$. Structures labeled as 30 and $60^{\circ}$ show changes with respect to the initial force distribution (close to $0^{\circ}$ ), whereas structures labeled as 90 and $120^{\circ}$ show changes with respect to $60^{\circ}$. (B) A network representation of changes in residue-wise forces at 60 and $120^{\circ}$, with cylinders scaled and colored according to magnitude and sign of the change, respectively. Four residues (Thr163, Glu188, Asp256, Arg260) important for transmitting force to the nucleotide-binding site are highlighted in orange, magenta, yellow and silver. The same reference force distributions are used as in panel A. Though the calculations neglect electrostatic screening by solvent molecules, it should have little impact on the description of interacting charges mostly buried in the protein interior.

$65-75^{\circ}$ (pre-ATP release) range as reference states (see Methods for details). The obtained values were then mapped onto the structures of individual $\beta$ subunits as color gradients.

As can be seen in Figure 4A, during the main rotary substep the force distribution in the nucleotide binding site region undergoes large changes, underscoring that, indeed, a considerable amount of mechanical energy is converted into elastic strain energy in the nucleotide-bound active site of $\beta_{\mathrm{TP}}$. In the network representation of changes in inter-residue forces (visualized in Figure $4 \mathrm{~B}$ as connecting cylinders colored and scaled according to the sign and magnitude of the change, respectively), the strongest connections are seen to involve the phosphate moiety and $\mathrm{Mg}^{2+}$ ion, with few nodes linked to the adenosine part of the nucleotide. This finding suggests that the accumulation of strain energy in the active site is at least partially due to the reshaping of the electrostatic environment surrounding the nucleotide-binding cavity. Combined with the free energy of ADP binding transmitted via the $\gamma$-mediated contact (see Figure 3), the energy stored in $\beta_{\mathrm{TP}}$ in the pre-ATPrelease state could provide the driving force required for the release of ATP, consistent with the model presented above. It is worth noting that, at the same point in the cycle, the force distribution in the catalytic site of $\beta_{\mathrm{DP}}$ changes only slightly, as would be expected for a subunit that remains almost unchanged over the whole $120^{\circ}$ synthetic cycle (Figure S5).

Yet, since the nucleotide-binding site is not immediately adjacent to the $\beta / \gamma$ interface where mechanical energy can be directly converted into conformational energy, the applied force has to propagate to the active site via a certain transmission network within the $\beta_{\mathrm{TP}}$ subunit. In Figure 4B, such a network representation of changes in pairwise inter-residue forces is shown for different rotation angles of the $\gamma$ shaft. Even though one cannot clearly distinguish a single path leading from the $\beta$ / $\gamma$ interface to the nucleotide-binding site, multiple connections that appear in the C-terminal part of $\beta_{\mathrm{TP}}$ can be further traced to the vicinity of the binding site in the central domain. As shown in Figure S6, a majority of these contributions result from large changes in Coulombic interactions that are insufficiently compensated by changes in steric ones, in line with the previously reported dominant role of electrostatics in mechanochemical coupling. ${ }^{22,24}$ Importantly, most of the pronounced changes in inter-residue forces are seen in the bottom part of the subunit which further highlights the relevance of the C-terminal domain for energy transmission. In particular, in helix 1, the salt bridge between Lys382 and Asp349/Asp352, enforced by hydrophobic contacts, directly connects the C-terminal domain and the central domain's main $\beta$-sheet, and, in helix 2, multiple connections transmit the strain to the back of the C-terminal domain. Through the $\beta$-sheet the above conformational changes further propagate toward the immediate vicinity of the nucleotide-binding site involving the contacts between the phosphate moiety and the GVGK motif of the P-loop, as well as between $\mathrm{Mg}^{2+}$ and its coordinating Thr163 residue. ${ }^{4,53}$ The interactions between the nucleotide and the surrounding charged residues, particularly Arg260, as well as Glu188 and Asp256 (involved in binding water molecules coordinated to $\mathrm{Mg}^{2+}$ ) are also perturbed (Figure 4B), as has also been observed previously. ${ }^{19}$ Moreover, the connections along helix 2 allow the force exerted on the Cterminal domain to be used to displace the lid-like element formed over the active site (red in Figure 1). Indeed, displacement of the lid-like loop can be observed over the whole $\gamma$ rotation cycle, increasing both conformational freedom and solvent accessibility of the adenine moiety, compared to the nucleotide tightly bound within $\beta_{\mathrm{DP}}$ (see Movie S3 and Figure S7).

To better characterize the energy transmission network within the catalytic subunit, we also investigated correlated changes in pairwise inter-residue forces by principal component analysis (PCA) (see Methods). Importantly, the use of 

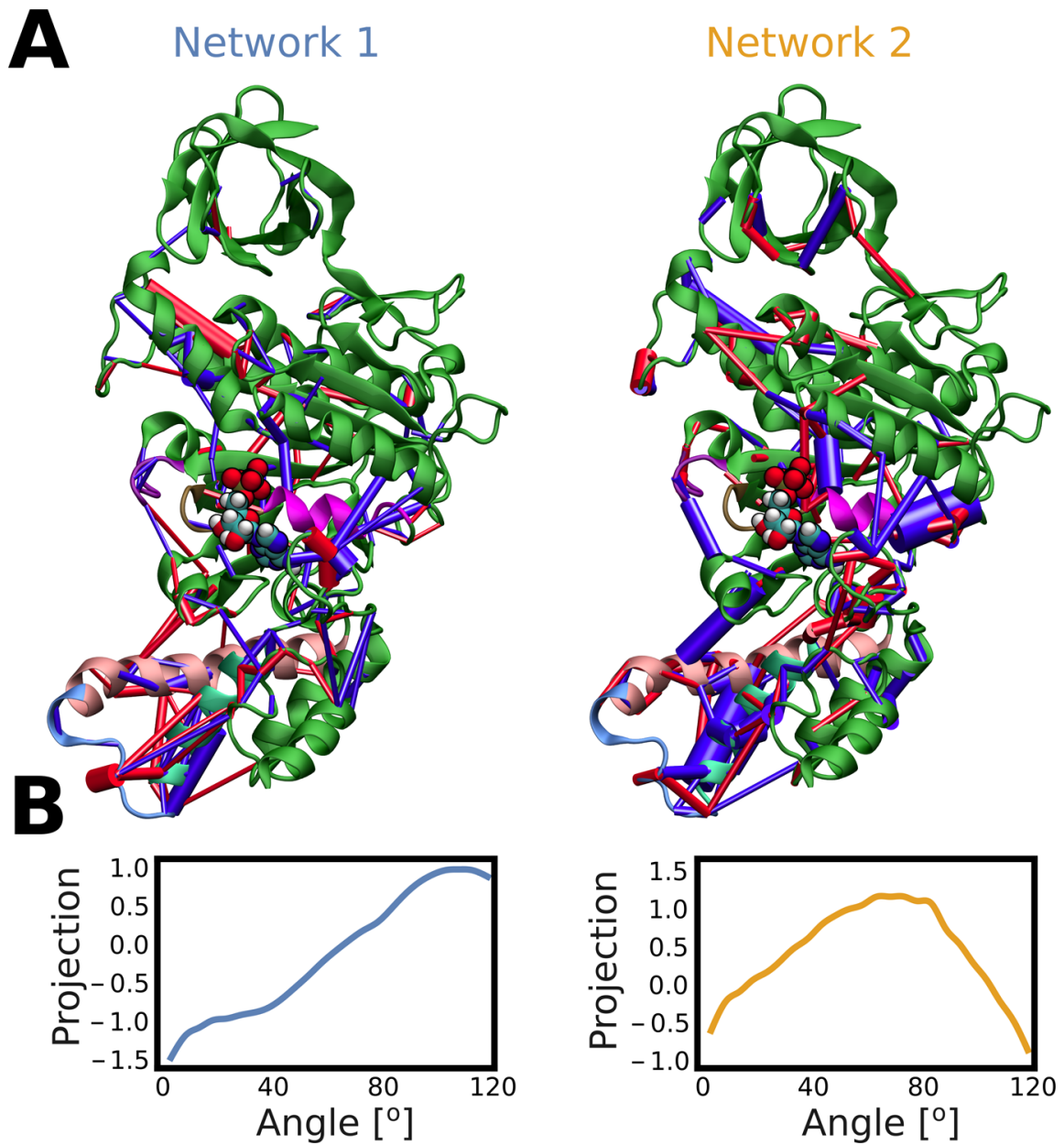

Figure 5. (A) Main allosteric networks involved in force transmission within the $\beta_{\mathrm{TP}}$ subunit, computed as the correlated changes in inter-residue forces from the force PCA (first two eigenvectors are presented, see also Figure S8). The cylinders are scaled and colored according to the magnitude and sign of the respective eigenvector component; for visualization purposes, only largest components are shown (see SI Methods). (B) Rotation-induced changes in inter-residue forces projected onto the two above eigenvectors.

correlated forces instead of correlated atomic positions has reportedly proven more sensitive in cases where allosteric transduction does not immediately result in large conformational changes, ${ }^{54}$ and should help identify actual force transmission networks while filtering out spurious signals arising from the correlated movements of, e.g., flexible protein termini. The two major networks, together describing $25 \%$ of variability in the force distribution, are shown in Figure 5A. Since the projections of actual force distributions shown in Figure 5B (see Figure S8 for comparison) correlate with features of the free energy profile, these networks can be assumed to represent two major modes of force transmission.

In network 1, the connections mostly propagate via helix 2 and the back of the C-terminal domain to accumulate in the vicinity of the lid-like loop, probably contributing to its gradual displacement (Figure S7 and Movie S3). In turn, network 2 involves larger contributions from the interactions between helix 1 and helix 2 , as well as helix 1 and the $\beta$-sheet adjacent to the nucleotide-binding site, providing a more direct connection to the active site. Network 2 also propagates-via helix 2-up to the HGG motif in the hinge region at the N-terminal end of helix B (colored in magenta in Figure 5), previously deemed crucial for the nucleotide binding-related conformational change of $\beta .^{36}$ Because both catch loops can directly transmit force from the $\gamma$ subunit (as indicated in Figure S9), force transmission from the $\gamma$-shaft appears to involve an extended cooperative network of interactions at the $\gamma / \beta$ interface rather than a single pathway. This idea of pathway heterogeneity provides a possible molecular explanation for mutagenesis studies which found that both interfacial regions, i.e., the cluster of three aspartates in catch 1 as well as the catch 2 loop (in particular, Glu395 of the DELSEED sequence and Arg408 in helix 2) are involved in the mechanochemical coupling in $F_{1}$ ATPase. ${ }^{34,55}$ The predominantly electrostatic character of the identified force transmission networks, shown in Figure S6 and Figure S10, also suggests how the coupling can be partially preserved despite significant structural changes ${ }^{56}$ but at the same time remain sensitive to alterations in local electrostatic properties. ${ }^{24}$ However, the actual balance between electrostatic and steric repulsion in $\gamma / \beta$ coupling is probably more convolved and requires further investigation, as highlighted by two studies that consistently found that abolishing the charge in the DELSEED region did not affect the torque produced by $\mathrm{F}_{1}$ during hydrolysis. ${ }^{57,58}$

A Checkpoint in the Pre-ATP-Release State Prevents Further Rotation Prior to Occupancy Change. Once the $70^{\circ}$ free energy minimum is reached, further rotation is strongly hampered for unchanged active site occupancies (see Figure 1C). While the torque distribution mapped onto the $\gamma$ subunit in Movie S1 suggests that this steep free energy rise results 
from a steric clash between the convex part of $\gamma$ shaft and the C-terminal domain of closed $\beta_{\mathrm{TP}}$, it is informative to examine the origin of this repulsive barrier in more detail. In Figure 4A, one can see that if $\gamma$ actually proceeds beyond the pre-ATPrelease state, much of the additional strain energy is further transferred to the vicinity of the binding site. Besides that, however, the rotation appears to be strongly opposed by electrostatic repulsion between the positively charged residues in the so-called "ionic track" ${ }^{59}$ on the protruding portion of $\gamma$ and in helix 2 of the C-terminal domain of $\beta_{\mathrm{TP}}$. Indeed, an analysis of interaction energies between individual structural elements of the catalytic subunits and the $\gamma$-shaft indicates that out of all contributions considered, the repulsion between helix 2 of $\beta_{\mathrm{TP}}$ and the protruding helix 1 of $\gamma$ shows the highest correlation with the free energy profile in the $80-120^{\circ}$ range and, at the same time, is largest in magnitude (Figure S11). To test this idea, we performed additional simulations in which we compared the spontaneous rotation of the $\gamma$-shaft, initiated at its final position at $120^{\circ}$, between the wild type $F_{1}$ and the mutant with all positively charged residues of helix 2 in $\beta_{\mathrm{TP}}$ (Lys401, Arg406, Arg408 and Lys406) replaced by alanines. From Figure S12 it can be seen that whereas in the wild type $\gamma$ immediately starts to rotate back toward the pre-ATP-release minimum, consistent with the free energy profile, no overall rotation is seen for the mutant during $200 \mathrm{~ns}$ of unrestrained simulation. This confirms a dominating role of the above electrostatic repulsion in stalling the motion of $\gamma$ at $\sim 70^{\circ}$, even though it is partially compensated by a number of contributions promoting further rotation, in particular, by the electrostatic attraction between the DELSEED sequence of $\beta_{\mathrm{E}}$ and the positively charged upper surface of the $\gamma$ globular domain (Figure S11). We thus propose that this interaction might act as a checkpoint mechanism, stalling further rotation of the $\gamma$ shaft until a concurrent substrate binding/product release event. Notably, similar checkpoint mechanisms ensuring completion of the conformational change prior to the irreversible step have been identified in other ATP-dependent motors such as myosin ${ }^{60,61}$ or the $\mathrm{ABC}$ transporter $\mathrm{TAP},{ }^{62}$ and are likely to exist in similar protein motors, e.g., V-ATPase or GTP-driven ribosomal factors. As these molecules generate force or torque by harnessing the excess free energy of irreversible reactions, such checkpoint mechanisms might have been frequently employed by evolution to ensure efficient chemomechanical coupling.

\section{CONCLUSIONS}

In this work, we present a thermodynamic, kinetic and mechanistic description of the main rotary substep in the synthetic cycle of the mammalian ATP synthase, providing a structural interpretation for recent single-molecule experiments. Our computed free energy profile is consistent with the previously observed stepwise character of $\gamma$-shaft rotation, showing a pronounced minimum yielding, under torque load, a metastable state at ca. $70^{\circ}$ and preceded by a steep free energy increase that represents and qualitatively explains the major free energy input in the ATP synthesis cycle. Indeed, both the $70^{\circ}$ step size as well as the associated free energy changes match measured magnitude and duration of the ATP-dependent rotary substep, ${ }^{12}$ indicating that the identified stable intermediate most likely corresponds to the elusive ATP-waiting state (or pre-ATP-release in synthesis direction). This result also corroborates that the typical X-ray structure captures the enzyme in the pre- $\mathrm{P}_{i}$-release state.
Notably, the calculated free energy changes-consistent with experimental rate constants to within $2-3 \mathrm{kcal} / \mathrm{mol}$ - appear to be fine-tuned to the overall cost of nucleotide occupancy change under cellular conditions. Our results thus support the model according to which the occupancy change needs to be mechanistically coupled in an anticooperative manner, in order to efficiently harness the energy of ADP binding to drive ATP release while avoiding energy dissipation.

Our simulations also provide structural insights into the mechanism that couples the $\gamma$-shaft rotation with the change in ligand affinities of the nucleotide binding sites. In particular, our conformational analysis shows that during the main substep of the catalytic cycle, the initially closed $\beta_{\text {TP }}$ subunit opens only partially, which challenges the prevailing view, but provides a thermodynamically consistent picture of the mechanochemical coupling, and finds support in previous computational studies. $^{19,42}$ As revealed by our force distribution analysis, instead of inducing an immediate global opening of $\beta_{\mathrm{TP}}$, the $\gamma$ shaft rotation causes local structural changes in the vicinity of the binding site involving the P-loop and charged residues. We propose that this accumulation of conformational energy in the binding site facilitates ATP release, which is subsequently followed by $\beta$ opening. The observed mechanism of energy conversion may well be a prototypic example for an interdomain communication that does not involve significant backbone rearrangements. ${ }^{63}$ We also find that this communication is mediated by a predominantly electrostatic force transmission network extended enough so that single residue mutations rarely compromise the robustness of mechanochemical coupling, as indeed reported in many mutagenesis studies. $^{24,57}$

Contrary to the conformationally intact $\beta_{\mathrm{TP}}$ subunit, $\beta_{\mathrm{E}}$ can be seen to spontaneously close during the $70^{\circ}$ substep from the open to a half-open conformation. As this partial transition preserves the $\gamma$-mediated contact between the C-terminal domains of $\beta_{\mathrm{E}}$ and $\beta_{\mathrm{TP}}$, it might actually be required for efficient coupling between ADP binding and ATP release when the motor stalls in the pre-ATP-release metastable state. Notably, this finding provides a possible explanation for an important prerequisite for energy efficient ATP synthesis, which so far has not been rationalized in structural terms: any further rotation of the $\gamma$-shaft in the metastable state prior to the occupancy change would waste free energy through irreversible downhill $\mathrm{ADP}$ binding and, hence, needs to be prevented. Our analysis of enthalpic profiles suggests that this stalling is due to the highly charged helix in the C-terminal domain of $\beta_{\mathrm{TP}}$ which in the closed, nucleotide-bound state directly opposes the corresponding charged region on the approaching $\gamma$-shaft. We propose that this interaction might effectively prevent further rotation until the occupancy change-induced opening of the $\beta_{\mathrm{TP}}$ subunit suppresses the clash, thus acting as a checkpoint that ensures the completion of a substrate binding/product release event. Such a mechanism might be a general feature of bionanomotors that would allow for highly efficient chemomechanical coupling under variable conditions.

\section{METHODS}

The initial configuration of the bovine $\mathrm{F}_{1}$-ATPase (PDB id 1E79) ${ }^{49}$ solvated with 87321 TIP4P water molecules at physiological ionic strength $(140 \mathrm{mM} \mathrm{NaCl})$ was assembled as described previously. ${ }^{42}$ The all-atom OPLS/AA force field ${ }^{64}$ was used for the protein, bound nucleotides and ions. In the $1 \mathrm{E} 79$ crystal structure, both $\beta$ subunits in the closed conformation $\left(\beta_{\mathrm{DP}}\right.$ and $\left.\beta_{\mathrm{TP}}\right)$ contain $\mathrm{ADP}$; therefore, to 
examine the effect of nucleotide on the energetics of rotation, a second system was prepared with the $\beta_{\mathrm{TP}}$-bound ADP replaced by ATP by using alchemical transformation. All MD simulations were carried out using Gromacs $4 .{ }^{65}$ See SI Methods for details concerning the simulation protocol and alchemical transformations.

All free energy profiles for the rotation of the $\gamma$-shaft, in the presence of $\mathrm{ADP}$ or ATP in $\beta_{\mathrm{TP}}$, were determined via umbrella sampling ${ }^{66}$ with the flexible-axis method ${ }^{67}$ used to impose a biasing potential in 25 windows spaced $5^{\circ}$ apart and spanning the angle range from 0 to $120^{\circ}$. The flexible-axis potential with a force constant of 35 $\mathrm{kJ} \cdot \mathrm{mol}^{-1} \cdot \mathrm{nm}^{-2}$ was applied to all $272 \mathrm{C}_{\alpha}$ atoms of the $\gamma$ subunit and the symmetry axis of the $\alpha_{3} \beta_{3}$ was taken as the rotation vector. To produce initial coordinates for the umbrella production runs, the system was first equilibrated for $500 \mathrm{~ns}$ at the initial angular position and then subjected to $1000 \mathrm{~ns}$ of enforced-rotation of the $\gamma$-shaft $(0 \rightarrow$ $120^{\circ}$ ), using the flexible-axis method (Movie S1). To model the immobilizing effect of the periphery stalk on $\alpha_{3} \beta_{3}$, all backbone atoms of six N-terminal residues of all $\beta$ subunits were harmonically restrained to their initial positions. In each of the windows the system was simulated for $400 \mathrm{~ns}$ yielding a total accumulated simulation time of $10 \mu \mathrm{s}$ for the $120^{\circ}$ rotation, and the free energy profiles were determined from the last $300 \mathrm{~ns}$ of the generated trajectories using the standard weighted histogram analysis method (WHAM) ${ }^{68}$ Error bars were estimated using Bayesian bootstrap approach. ${ }^{69}$

The force distribution analysis (FDA) was performed using the FDA module implemented in Gromacs 4.5.3. ${ }^{52,70}$ Changes in force distributions were calculated with respect to reference distributions in the pre- $\mathrm{P}_{i}$ release state and the ATP-dependent state to observe the accumulation of strain in the rotary substeps with spatial resolution. For the purpose of visualization, the obtained changes in residue-wise forces were either shown as cylinders or mapped onto the structure of the catalytical subunit as color code. Correlated charges in interresidue forces were extracted using principal component analysis (PCA), and the resulting force propagation networks were shown in the cylinder representation (see SI Methods).

\section{ASSOCIATED CONTENT}

\section{S Supporting Information}

The Supporting Information is available free of charge on the ACS Publications website at DOI: 10.1021/jacs.6b11708.

Detailed description of methods; thermodynamic cycle for nucleotide exchange; 13 supplementary figures, including force distribution analysis and intersubunit interactions energies (PDF)

Movie S1 (MPG)

Movie S2 (MPG)

Movie S3 (MPG)

\section{AUTHOR INFORMATION}

\section{Corresponding Author}

*hgrubmu@gwdg.de

\section{ORCID}

Miłosz Wieczór: 0000-0003-4990-8629

Notes

The authors declare no competing financial interest.

\section{ACKNOWLEDGMENTS}

This work was supported by the EU Nanomot (NEST 029084) and ESF Eurocores Nanocell projects and by the Polish Ministry of Science and Higher Education (Project No. IP2012 03472). This research was supported in part by PL-Grid Infrastructure and the TASK computational center.

\section{REFERENCES}

(1) Boyer, P. Annu. Rev. Biochem. 1997, 66, 717-749.
(2) Kinosita, K., Jr; Adachi, K.; Itoh, H. Annu. Rev. Biophys. Biomol. Struct. 2004, 33, 245-268.

(3) Junge, W.; Sielaff, H.; Engelbrecht, S. Nature 2009, 459, 364370 .

(4) Abrahams, J.; Leslie, A.; Lutter, R.; Walker, J. Nature 1994, 370, 621-628.

(5) Noji, H.; Yasuda, R.; Yoshida, M.; Kinosita, K. Nature 1997, 386, 299-302.

(6) Sabbert, D.; Engelbrecht, S.; Junge, W. Proc. Natl. Acad. Sci. U. S. A. 1997, 94, 4401-4405.

(7) Yasuda, R.; Noji, H.; Kinosita, K.; Yoshida, M. Cell 1998, 93, 1117-1124.

(8) Börsch, M.; Diez, M.; Zimmermann, B.; Reuter, R.; Gräber, P. FEBS Lett. 2002, 527, 147-152.

(9) Watanabe, R.; Tabata, K. V.; Iino, R.; Ueno, H.; Iwamoto, M.; Oiki, S.; Noji, H. Nat. Commun. 2013, 4, 1631.

(10) Yasuda, R.; Noji, H.; Yoshida, M.; Kinosita, K.; Itoh, H. Nature 2001, 410, 898-904.

(11) Shimabukuro, K.; Yasuda, R.; Muneyuki, E.; Hara, K. Y.; Kinosita, K.; Yoshida, M. Proc. Natl. Acad. Sci. U. S. A. 2003, 100, 14731-14736.

(12) Suzuki, T.; Tanaka, K.; Wakabayashi, C.; Saita, E.-i.; Yoshida, M. Nat. Chem. Biol. 2014, 10, 930-936.

(13) Watanabe, R.; Iino, R.; Noji, H. Nat. Chem. Biol. 2010, 6, 814820.

(14) He, Z.-H.; Bottinelli, R.; Pellegrino, M. A.; Ferenczi, M. A.; Reggiani, C. Biophys. J. 2000, 79, 945-961.

(15) Moore, K.; Lohman, T. M. Biophys. J. 1995, 68, 180 S.

(16) Toyabe, S.; Watanabe-Nakayama, T.; Okamoto, T.; Kudo, S.; Muneyuki, E. Proc. Natl. Acad. Sci. U. S. A. 2011, 108, 17951-17956.

(17) Wang, H.; Oster, G. Nature 1998, 396, 279-282.

(18) Oster, G.; Wang, H. Biochim. Biophys. Acta, Bioenerg. 2000, $1458,482-510$.

(19) Böckmann, R. A.; Grubmüller, H. Nat. Struct. Biol. 2002, 9, 198-202.

(20) Dittrich, M.; Hayashi, S.; Schulten, K. Biophys. J. 2004, 87, 2954-2967.

(21) Adachi, K.; Oiwa, K.; Nishizaka, T.; Furuike, S.; Noji, H.; Itoh, H.; Yoshida, M.; Kinosita, K. Cell 2007, 130, 309-321.

(22) Mukherjee, S.; Warshel, A. Proc. Natl. Acad. Sci. U. S. A. 2011, $108,20550-20555$.

(23) Okazaki, K.; Hummer, G. Proc. Natl. Acad. Sci. U. S. A. 2013, 110, 16468-16473.

(24) Mukherjee, S.; Warshel, A. Proc. Natl. Acad. Sci. U. S. A. 2015, $112,2746-2751$.

(25) Cherepanov, D.; Mulkidjanian, A.; Junge, W. FEBS Lett. 1999, 449, 1-6.

(26) Ernst, S.; Düser, M. G.; Zarrabi, N.; Dunn, S. D.; Börsch, M. Biochim. Biophys. Acta, Bioenerg. 2012, 1817, 1722-1731.

(27) Sielaff, H.; Rennekamp, H.; Wächter, A.; Xie, H.; Hilbers, F.; Feldbauer, K.; Dunn, S.; Engelbrecht, S.; Junge, W. Proc. Natl. Acad. Sci. U. S. A. 2008, 105, 17760-17765.

(28) Czub, J.; Grubmüller, H. Proc. Natl. Acad. Sci. U. S. A. 2011, 108, $7408-7413$

(29) Okazaki, K.-i.; Hummer, G. Proc. Natl. Acad. Sci. U. S. A. 2015, 112, 10720-10725.

(30) Miwa, K.; Yoshida, M. Proc. Natl. Acad. Sci. U. S. A. 1989, 86, 6484-6487.

(31) Furuike, S.; Hossain, M.; Maki, Y.; Adachi, K.; Suzuki, T.; Kohori, A.; Itoh, H.; Yoshida, M.; Kinosita, K., Jr Science 2008, 319, 955.

(32) Senior, A. E.; Nadanaciva, S.; Weber, J. Biochim. Biophys. Acta, Bioenerg. 2002, 1553, 188-211.

(33) Masaike, T.; Koyama-Horibe, F.; Oiwa, K.; Yoshida, M.; Nishizaka, T. Nat. Struct. Mol. Biol. 2008, 15, 1326-1333.

(34) Arsenieva, D.; Symersky, J.; Wang, Y.; Pagadala, V.; Mueller, D. M. J. Biol. Chem. 2010, 285, 36561-36569.

(35) Watanabe, R.; Koyasu, K.; You, H.; Tanigawara, M.; Noji, H. Biophys. J. 2015, 108, 1144-1152. 
(36) Masaike, T.; Mitome, N.; Noji, H.; Muneyuki, E.; Yasuda, R.; Kinosita, K.; Yoshida, M. J. Exp Biol. 2000, 203, 1-8.

(37) Nakanishi-Matsui, M.; Kashiwagi, S.; Ubukata, T.; IwamotoKihara, A.; Wada, Y.; Futai, M. J. Biol. Chem. 2007, 282, 20698-20704.

(38) Shen, H.; Yao, B. Y.; Mueller, D. M. J. Biol. Chem. 1994, 269, 9424-8.

(39) Strajbl, M.; Shurki, A.; Warshel, A. Proc. Natl. Acad. Sci. U. S. A. 2003, 100, 14834-14839.

(40) Pu, J.; Karplus, M. Proc. Natl. Acad. Sci. U. S. A. 2008, 105, 1192.

(41) Nam, K.; Pu, J.; Karplus, M. Proc. Natl. Acad. Sci. U. S. A. 2014,

111, 17851-17856.

(42) Czub, J.; Grubmüller, H. J. Am. Chem. Soc. 2014, 136, 69606968.

(43) Ito, Y.; Oroguchi, T.; Ikeguchi, M. J. Am. Chem. Soc. 2011, 133, 3372-3380.

(44) Mukherjee, S.; Bora, R. P.; Warshel, A. Q. Rev. Biophys. 2015, 48, 395-403.

(45) Astumian, R. D.; Mukherjee, S.; Warshel, A. ChemPhysChem 2016, 17, 1719-1741.

(46) Singharoy, A.; Chipot, C.; Moradi, M.; Schulten, K. J. Am. Chem. Soc. 2017, 139, 293-310.

(47) Martin, J. L.; Ishmukhametov, R.; Hornung, T.; Ahmad, Z.; Frasch, W. D. Proc. Natl. Acad. Sci. U. S. A. 2014, 111, 3715-3720.

(48) Turina, P.; Samoray, D.; Gräber, P. EMBO J. 2003, 22, 418426.

(49) Gibbons, C.; Montgomery, M.; Leslie, A.; Walker, J. Nat. Struct. Biol. 2000, 7, 1055-1061.

(50) Adachi, K.; Oiwa, K.; Yoshida, M.; Nishizaka, T.; Kinosita, K., Jr Nat. Commun. 2012, 3, 1022.

(51) Yagi, H.; Kajiwara, N.; Iwabuchi, T.; Izumi, K.; Yoshida, M.; Akutsu, H. J. Biol. Chem. 2009, 284, 2374-2382.

(52) Stacklies, W.; Seifert, C.; Graeter, F. BMC Bioinf. 2011, 12, 1-5.

(53) Menz, R.; Walker, J.; Leslie, A. Cell 2001, 106, 331-341.

(54) Seifert, C.; Gräter, F. Biophys. J. 2012, 103, 2195-2202.

(55) Chen, X. J.; Clark-Walker, G. D. EMBO J. 1995, 14, 3277.

(56) Chiwata, R.; Kohori, A.; Kawakami, T.; Shiroguchi, K.; Furuike, S.; Adachi, K.; Sutoh, K.; Yoshida, M.; Kinosita, K. Biophys. J. 2014, 106, 2166-2174.

(57) Hara, K. Y.; Noji, H.; Bald, D.; Yasuda, R.; Kinosita, K.; Yoshida, M. J. Biol. Chem. 2000, 275, 14260-14263.

(58) Tanigawara, M.; Tabata, K.; Ito, Y.; Ito, J.; Watanabe, R.; Ueno, H.; Ikeguchi, M.; Noji, H. Biophys. J. 2012, 103, 970-978.

(59) Ma, J.; Flynn, T.; Cui, Q.; Leslie, A.; Walker, J.; Karplus, M. Structure 2002, 10, 921-931.

(60) Ito, K.; Uyeda, T. Q. P.; Suzuki, Y.; Sutoh, K.; Yamamoto, K. J. Biol. Chem. 2003, 278, 31049-31057.

(61) Kad, N. M.; Trybus, K. M.; Warshaw, D. M. J. Biol. Chem. 2008, 283, 17477-17484.

(62) Grossmann, N.; Vakkasoglu, A. S.; Hulpke, S.; Abele, R.; Gaudet, R.; Tampé, R. Nat. Commun. 2014, 5, 5419.

(63) Tsai, C.-J.; Del Sol, A.; Nussinov, R. J. Mol. Biol. 2008, 378, 111.

(64) Kaminski, G. A.; Friesner, R. A.; Tirado-Rives, J.; Jorgensen, W. L. J. Phys. Chem. B 2001, 105, 6474-6487.

(65) Hess, B. J. Chem. Theory Comput. 2008, 4, 116-122.

(66) Torrie, G. M.; Valleau, J. P. J. Comput. Phys. 1977, 23, 187-199.

(67) Kutzner, C.; Czub, J.; Grubmüller, H. J. Chem. Theory Comput. 2011, 7, 1381-1393.

(68) Kumar, S.; Bouzida, D.; Swendsen, R. H.; Kollman, P. A.; Rosenberg, J. M. J. Comput. Chem. 1992, 13, 1011-1021.

(69) Hub, J. S.; De Groot, B. L.; Van Der Spoel, D. J. Chem. Theory Comput. 2010, 6, 3713-3720.

(70) Xiao, S.; Stacklies, W.; Cetinkaya, M.; Markert, B.; Gräter, F. Biophys. J. 2009, 96, 3997-4005. 